\title{
Correlation between fixation systems elasticity and bone tunnel widening after ACL reconstruction
}

\author{
Nicola Giorgio ${ }^{1}$ \\ Lorenzo Moretti ${ }^{1}$ \\ Paolo Pignataro² \\ Massimiliano Carrozzo ${ }^{1}$ \\ Giovanni Vicenti ${ }^{1}$ \\ Biagio Moretti ${ }^{1}$ \\ 1 Department of Basic Medical Sciences, \\ Neurosciences and Sensory Organs, UO \\ Orthopaedics and Traumatology, Azienda Ospe- \\ daliero Universitaria "Policlinico di Bari", Bari, Italy \\ 2 UO University Diagnostic Radiology, Azienda \\ Ospedaliero Universitaria "Policlinico di Bari", \\ Bari, Italy
}

Corresponding author:

Giovanni Vicenti

Department of Basic Medical Sciences,

Neurosciences and Sensory Organs, UO

Orthopaedics and Traumatology, Azienda Ospe-

daliero Universitaria "Policlinico di Bari"

Piazza G. Cesare 11

70124 Bari, Italy

E-mail: dott.gvicenti@gmail.com

\section{Summary}

Background: Femoral and tibial tunnel widening (TW) after ACL reconstruction is a phenomenon increasing talk in the literature. It is underlying biological and mechanical causes.

Objective: The aim of this study was to evaluate the relationship between bone tunnel enlargement and two different ACL fixation systems.

Patients and Methods: 40 patient underwent ACL reconstruction with hamstring; randomly divided into group A with 20 patients treated with stiff systems (femoral Rigidfix and tibial interference screw), and into group B, with 20 patients treated with morel elastic system (femoral and tibial Tight-rope). Evaluated postoperatively with knee MRI at 40 days, 3 months, 6 months to measure bone tunnel diameters widening.

Results: At $\mathbf{4 0}$ days tunnel widening between two groups shows no statistically difference. At 3 months postoperatively, femoral bone tunnel widening amounted on average to $1.84 \mathrm{~mm}$ in middle of tunnel and $1 \mathrm{~mm}$ at the mouth in joint in group A, and respectively $3.2 \mathrm{~mm}$ and $2.5 \mathrm{~mm}$ in group $B(p<0.05)$. Tibial tunnel widening was 1.24 $\mathrm{mm}$ at the mouth in joint and $1.3 \mathrm{~mm}$ in middle in group $A$ and respectively $2.26 \mathrm{~mm}$ and $2.43 \mathrm{~mm}$ in group $B(p<0.05)$.

At 6 months femoral tunnel widening amounted on average to $2.45 \mathrm{~mm}$ in middle and $1.35 \mathrm{~mm}$ at the mouth in joint in group $A$ and respectively 3.5 $\mathrm{mm}$ and $2.7 \mathrm{~mm}$ in group $B(p<0.01)$. Tibial tunnel widening amounted on average to 1.27 at mouth in joint and $1 \mathrm{~mm}$ in middle of tunnel in group $A$ and respectively $2.6 \mathrm{~mm}$ and $2.3 \mathrm{~mm}$ in group $B$ $(p<0.01)$.

Conclusions: This study results suggest elastic fixation system increases bone tunnel enlargement after $A C L$ reconstruction with hamstring without correlation with worse clinical performance.

Level of evidence: IV.

KEY WORDS: anterior cruciate ligament reconstruction, graft fixation methods, tunnel widening, clinical outcome.

\section{Introduction}

There are many graft materials available for the treatment of an ACL-deficient knee. ACL reconstruction in the last 20 years has seen many changes on best graft to use (BTB, hamstring, synthetic devices), on surgical technique (transtibial, anteromedial, overthe-top, single or double bundle, all-inside technique). Some studies reported as one of the complications the a gradual widening of the drilled femoral and tibial tunnels in ACL-reconstructed knees ${ }^{1-4}$. There seems to be a significantly greater risk of TW when using hamstring grafts compared with patellar tendon bone graft $^{5}$. The bone tunnel remodeling is significantly different with regards to the fixation method, graft and tunnel diameter 6 . Many studies describe the mechanical explanations for TW as the windshield-wiper effect and the bungee cord effect ${ }^{1}$ that are based on the hypothesis that the graft slides inside the tunnel during the motion of the knee, in either a longitudinal or a transverse direction ${ }^{5}$ (Fig. $1 \mathrm{a}, \mathrm{b}$ ).

Comparative studies between vary fixation systems are encumbered by intrinsic limits as they often compare systems with biomechanical characteristics and engineering philosophy completely different, implanted on bone with density and biomechanical property far away from those young typical patient that under- 


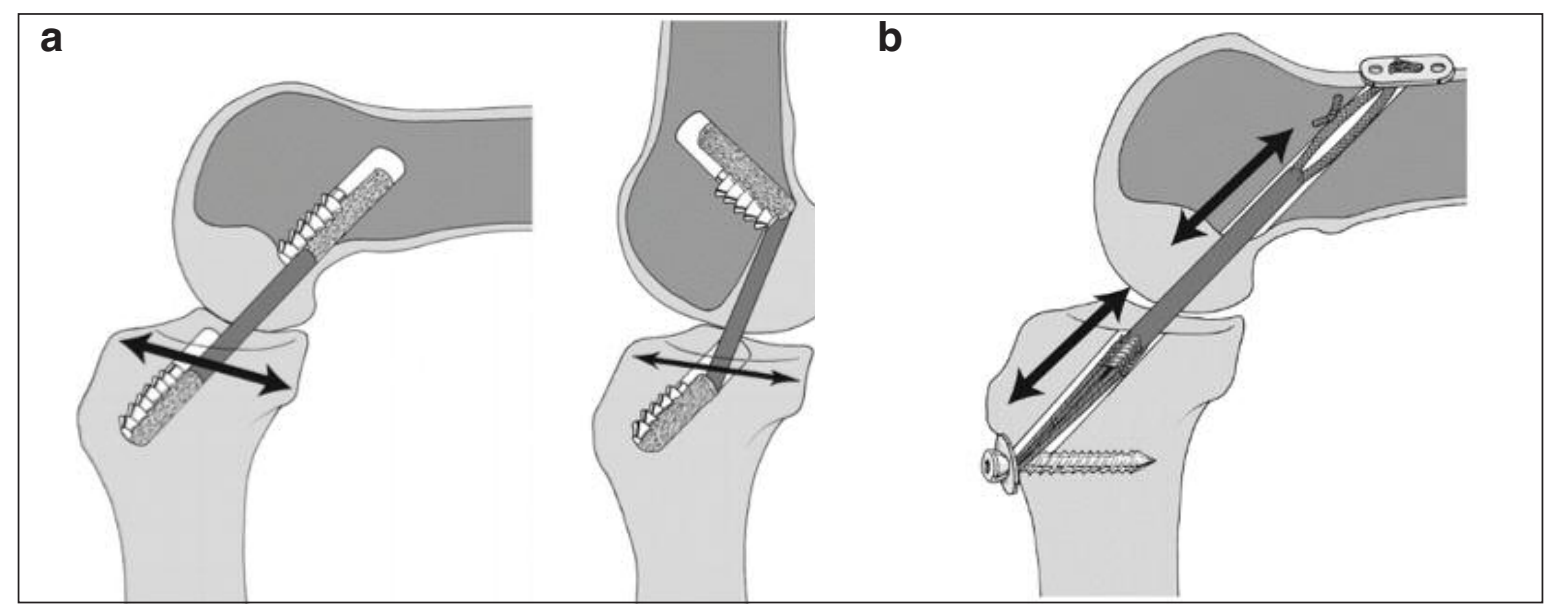

Figure 1a, b. a) Windshield wiper effect. b) -Bungee cord effect.

went $A C L$ reconstruction (bone to elderly or donor animal $)^{7,8}$. The direction of force application in tests to evaluate ultimate tensile load or pull-out strength is only in one direction, while other movements are excluded; or femoral and tibial fixation systems are tested separately, forgiving the complexity of femoral and tibial bone-graft-device system ${ }^{9}$.

Laboratory studies suggest grafts homogeneity in terms of stiffness and ultimate tensile load compared to native ACL; whereas are not so clear the mechanicals property devices should have in the "system".

Native ACL has an ultimate tensile load of $2160 \mathrm{~N}$ and a stiffness of $242 \mathrm{~N} / \mathrm{mm}^{10}$.

The current substitutes are all able to provide adequate values at time zero: $2977 \mathrm{~N}$ e $455 \mathrm{~N} / \mathrm{mm}$ for patellar tendon ${ }^{11} ; 4140 \mathrm{~N}$ e $807 \mathrm{~N} / \mathrm{mm}$ for hS quadrupled $2353 \mathrm{~N}$ e $326 \mathrm{~N} / \mathrm{mm}$ for quadriceps tendon ${ }^{12}$.

About fixation systems, literature evidences that less than $500 \mathrm{~N}$ are adequate for early rehabilitation protocol and daily living.

Fixation systems divide into three main groups: compression, expansion and suspension.

Compression systems use interference screw. Last upgrades made this system more reliable, because less aggressive screw threads reduced tendon tear risk. However, fixation with this device is affected by bone density, direction of insertion, diameter (increasing it improves the strength), length, material and screw design.

In some studies, it has been reported that resorbable screw average ultimate tensile strength and stiffness (ranging from 341 and $576 \mathrm{~N}$, and from 29 and 252 $\mathrm{N} / \mathrm{mm}$ respectively) is higher than metal screw (ranging from 240 and $617 \mathrm{~N}$, and 71 and $257 \mathrm{~N} / \mathrm{mm}$ respectively). In other studies, there was no statistical difference in stability and in the incidence of complications. Graft interference fixation perform an anatomical fixation near the joint and improve isometric implant ${ }^{13}$.

Expansion fixation, is made up by one or more transverse pins that pass-through graft, properly prepared, leading graft swelling in bone tunnel. This system depends by primary graft press-fit, bone density, right pin position. Eccentric pin implant would lead an unbalanced load distribution, compromising graft integration and system failure.

Suspension fixation is indirect system that hang graft on sutures or polyester connected to metal button (e.g. endobutton) with ultimate tensile load of $850 \pm 189.8 \mathrm{~N}$ and stiffness of $112.5 \pm 9.7 \mathrm{~N} / \mathrm{mm}$; or completely metal systems (e.g. Swing Bridge) with ultimate tensile load of $1359 \pm 214.1 \mathrm{~N}$ and stiffness of $162.6 \pm 45.8 \mathrm{~N} / \mathrm{mm}$. Device is implanted on antero-lateral femoral cortex or outside tibial tunnel.

Possible problems of this technique can be lateral skin incision, uncomfortable presence of foreign body that irritates antero-lateral soft tissues bringing to metal removal, difficulties in removing the system, bone loss management in revision surgery ${ }^{14}$.

The Endobutton ${ }^{\circledR}$ has an easy technique, fast and reproducible. Its disadvantage is relating to longitudinal and sagittal graft movements and the concomitant synovial fluid entrance in the tunnels. These movements create shear forces that could delay the graft healing and comport the tunnel widening. It has been shown that loads between $100 \mathrm{~N}$ and $300 \mathrm{~N}$, induce $0.3 \mathrm{~mm}$ graft motion respect tunnel. In our study was used Tight-rope system, an upgrade of the concept of endobutton, that can intraoperatively adapt polyester loop length to improve tension graft.

Cortico-cancellous suspension fixation use a transcondilar device that crosses bone tunnel and graft overhangs it with orthogonal pullout forces. Transfix ${ }^{\circledR}$ (ultimate tensile load $1469.7 \pm 315.5 \mathrm{~N}$; stiffness $206.7 \pm 9.7 \mathrm{~N} / \mathrm{mm}$ ), Bio-transfix ${ }^{\circledR}$ (ultimate tensile load 1491.6 \pm 87.6 ; stiffness $210.1 \pm 67.9)$ and Sling Shot Crosspin (Mitek) belong to this class. Mechanical property depends on bone density, length of the lever arms respect to suspension point of graft. Potential risks are saphenous and peroneal nerve injury, lateral collateral injury, iliotibial band syndrome that in some studies get to $16 \%$ of incidence ${ }^{15,16}$. 
Optimal graft positioning for ACL reconstruction is being thoroughly investigated in the literature ${ }^{17}$ and mechanical properties of fixation systems are overall still inadequate; therefore, still represent the weak link: proper stiffness would restore normal knee kinematic on load and reduce graft motion in the tunnel. For integration of a grafted tendon with bone, the mechanical environment at the tendon-bone interface is one of the major contributory factors ${ }^{18}$.

It is easy to think that graft motion is caused by low stiffness systems as extra cortical fixation (far from the origin of bone tunnel) and hamstring ${ }^{19}$. We remember that the graft necrosis usually observed in the initial two weeks of the healing process is averted by inducing gaps into the tendon graft prior to $A C L$ reconstruction ${ }^{20}$.

This phenomenon is determined by the position of the tunnel in reciprocal movement between them (windshield wiper effect), and by implant elasticity (bungee cord effect).

An excessive movement can prejudice graft healing, induce bone tunnel widening (evidenced with BtB and hS) and affect knee biomechanics ${ }^{21}$.

Ideal fixation should minimize graft motion, prevent synovial fluid access in bone tunnel and increase anterior stability of the knee ${ }^{22}$. Bone loss following surgical reconstructions of the ACL is mostly not caused by the immobilisation before and after the surgery, but rather due to limitations of the functional result with regards to tendon stiffness and muscle force ${ }^{23}$. Most of the studies confirm that bone tunnel enlargement is not connected to worse clinical performances ${ }^{21,24}$. The objective of our study is to understand correlation between different stiffness fixation systems and femoral and tibial tunnel widening.

\section{Patient and method}

We conducted a prospective study ${ }^{25}$ on patients with ACL-deficient knees. From April 2014 to December 2015, one surgeon (L.M.) performed 73 ACL reconstruction using double Semitendinosus and Gracilis Tendon (ST/G). Inclusion criteria were: symptomatic instability due to ACL deficiency, age lesser than 60 years old, no metabolic bone disease. Patients with associated posterior cruciate ligament injuries, previous knee surgeries were excluded.

Forty patients with age between 17 and 51 years, $80 \%$ males and $20 \%$ females, were included in the study. They were randomly divided into group A with 20 patients treated with "stiff" systems (femoral Rigidfix and tibial reabsorbable interference screw), and into group B, with 20 patients treated with more elastic system (femoral and tibial Tight-rope).

A standardized post-operative and rehabilitation protocol was used. Full weight bearing was allowed immediately after surgery. A brace allowing full range of motion and crutches were used during the first 15 postoperative days.

Post-operative follow-up was performed with MRI at 40 days, 3 months, 6 months in the same radiological institute using MRI Siemens 1.5 tesla machine, with dedicated knee coil.

The imagin was performed in supine position after routine patient preparation. All patients were imaged in sagittal and coronal STIR (TR: $4100 \mathrm{~ms}$; TE: 30 ms; Slice thickness $3 \mathrm{~mm}$ ), sagittal PD (TR: $2000 \mathrm{~ms}$; TE: $17 \mathrm{~ms}$; Slice thickness $3 \mathrm{~mm}$ ) Sagittal and axial T2 (TR: $3000 \mathrm{~ms}$; TE: $90 \mathrm{~ms}$, Slice thickness $3 \mathrm{~mm}$ ). A third operator (P. P.) acquired femoral and tibial tunnel diameter measure, in middle and in mouth in joint, in MRI scans. The IKDC classification and Lyslholm scores were recorded for each patient. Statistical analysis was performed using $\mathrm{R}$ Studio (Version 1.0.40). The comparison of quantitative data and the paired comparison of normally distributed parameters were performed using the Student $t$ test.

\section{Results}

At 40 days tunnel widening between two groups shows no statistically difference. At 3 months postoperatively, femoral bone tunnel widening amounted on average to $1.84 \mathrm{~mm}$ in middle of tunnel and $1 \mathrm{~mm}$ at the mouth in joint in group $A$, and respectively 3.2 $\mathrm{mm}$ and $2.5 \mathrm{~mm}$ in group $B(p<0.05)$. Tibial tunnel widening was $1.24 \mathrm{~mm}$ at the mouth in joint and 1.3 $\mathrm{mm}$ in middle in group $A$ and respectively $2.26 \mathrm{~mm}$ and $2.43 \mathrm{~mm}$ in group $B(p<0.05)$ (Fig. 2 a-d).

At 6 months femoral tunnel widening amounted on average to $2.45 \mathrm{~mm}$ in middle and $1.35 \mathrm{~mm}$ at the mouth in joint in group $A$ and respectively $3.5 \mathrm{~mm}$ and 2.7 $\mathrm{mm}$ in group $B(p<0.01)$. Tibial tunnel widening amounted on average to 1.27 at mouth in joint and 1 $\mathrm{mm}$ in middle of tunnel in group $A$ and respectively 2.6 $\mathrm{mm}$ and $2.3 \mathrm{~mm}$ in group $\mathrm{B}(\mathrm{p}<0.01)(T a b . \mathrm{l})$.

Furthermore, bone tunnel enlargement on suspension fixation appears greater on femoral side than in tibial side. No early and late complications were detected.

\section{Discussion}

In our study we compared 2 different fixation systems, each with its characteristics and we found that the TW appear to be influenced by the intrinsic mechanical properties of the implants. The use of a more elastic fixation systems (Tight Rope) is associated with more laxity and determinate a bigger tunnel widening.

In literature number of studies about tunnel widening after ACL reconstruction has a little but increasing trend in last ten years.

Other Authors studied correlation between different fixation systems and bone tunnel enlargement. Many TC, MRI, X-ray studies suggest that suspension systems (e.g. endobutton) lead to bone tunnel increase respect other concepts of fixation ${ }^{26,27}$. Common element of these studies is that tunnel enlargement is not connected to worse clinical performances.

Our results assessment shows no statistical difference at 40 days. A possible hypothesis comes from a 


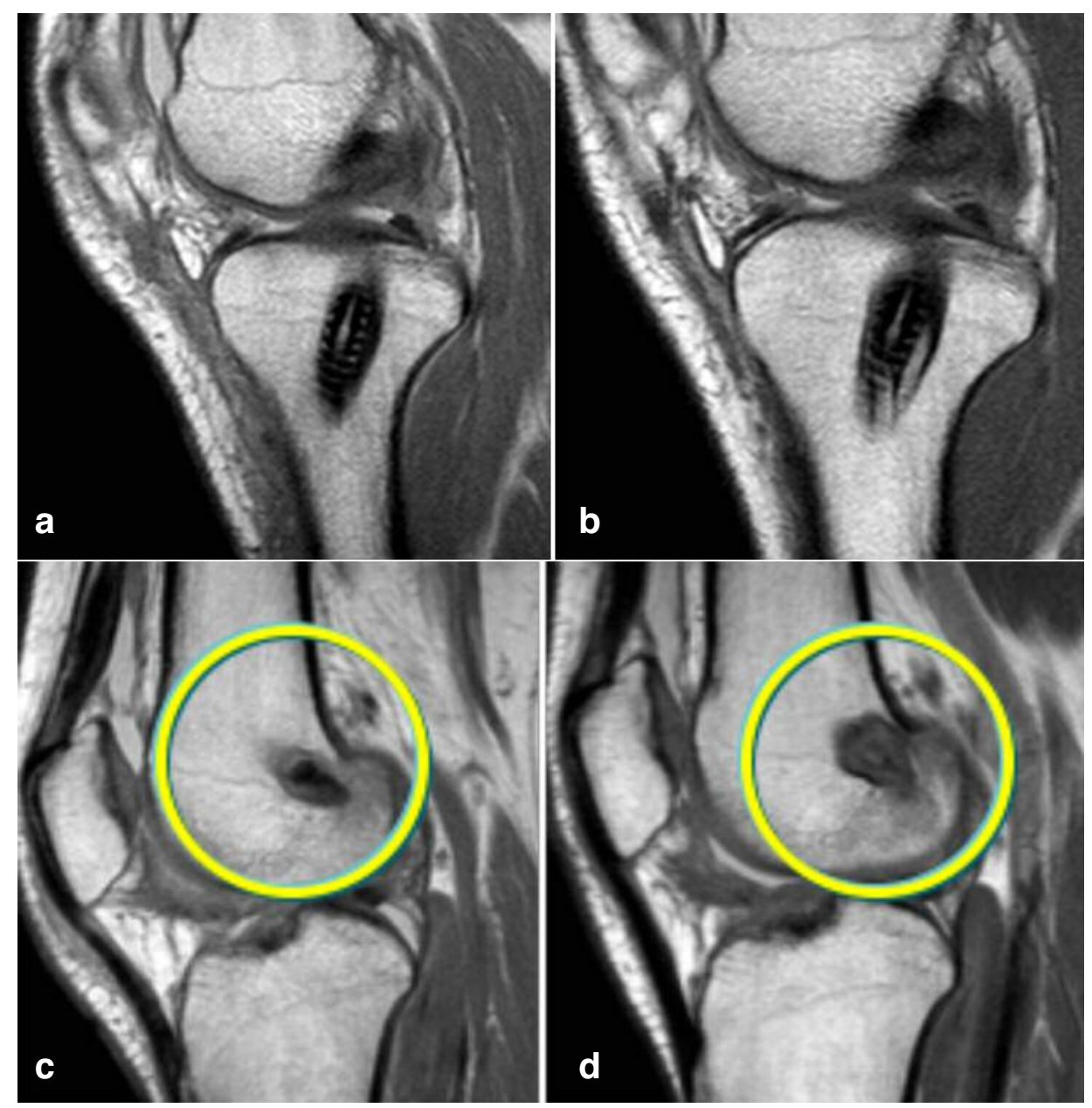

Figure 2 a-d. a) MRI at 40 days. Tibial interference screw. b) MRI at 6 months. Tibial interference screw. c) $\mathrm{MRI}$ at 40 days. Femoral tight-rope. d) MRI at 40 days. Femoral tight-rope.

Table I. Tunnel widening at 6 months in group A an B.

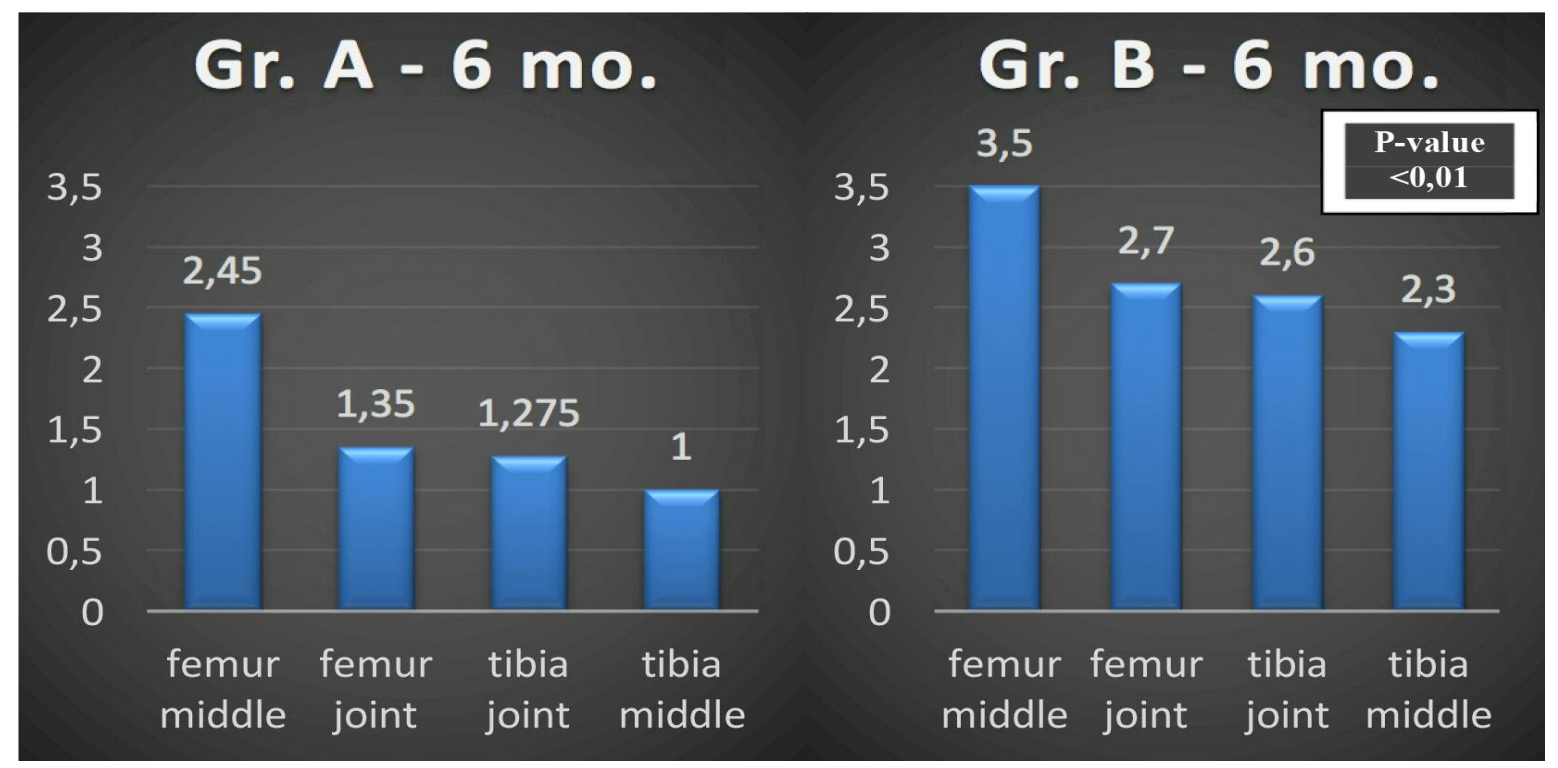


recent study on KSSTA, in which have been evaluated biomechanical factors, morphology, and immunohistochemistry in murine model underwent on ACL reconstruction. It shows that peri-implant bone resorbtion a time-depending process, sustained by metalloprotease and CD68+ cells within 6 weeks ${ }^{28}$.

Therefore is possible to state, as for graft healing process $^{29}$, that femoral and tibial bone tunnel enlargement comes from a balance between biological factors and biomechanical factors. Nowadays radiological imaging upgrade can combine morphology and metabolism of ligaments like in PET-MRI, and some studies suggest that biological rearrangement, after ACL reconstruction, continues up to 24 months postoperatively ${ }^{30}$.

However, in literature there are many attempt in order to modify tunnel widening, either from biological side, with the controversial use of $\mathrm{PRP}^{31,32}$, preserving $A C L$ remnant ${ }^{33}$, hybrid graft ${ }^{34}$, alendronate ${ }^{35}$, manual drilling to reduce termical stress on bone ${ }^{36}$; either from biomechanical side, improving tunnel position ${ }^{37}$ and fixation systems stiffness. All these studies are conducted on little patient cohorts or on animal mod$\mathrm{el}$, therefore it is desirable our little clinical experience would continue to also understand biological factors combined with biomechanical element.

In the current study, only two different femoral fixation implants had been evaluated. This is one of the limitations of the study. Different positioning of the tunnel, correlation between conventional radiography and CT scan and loop length could be added into consideration in a future study.

\section{Conclusion}

From the experience of this study, we can conclude that ACL reconstruction with hamstring is subjected to femoral and tibial bone tunnel widening, the most among the use of fixation systems more elastic. Literature revision unanimously suggests that this phenomenon do not correlate to lower clinical performances, at least in the short term.

\section{Conflict of interest}

The Authors certify that they have no affiliations with or involvement in any organization or entity with any financial interest (such as honoraria; educational grants; participation in speakers' bureaus; membership, employment, consultancies, stock ownership, or other equity interest; and expert testimony or patentlicensing arrangements), or non-financial interest (such as personal or professional relationships, affiliations, knowledge or beliefs) in the subject matter or materials discussed in this manuscript.

\section{References}

1. L'Insalata JC, Klatt B, Fu FH, Harner CD. Tunnel expansion following anterior cruciate ligament reconstruction: a comparison of hamstring and patellar tendon autografts. Knee Surg Sports Traumatol Arthrosc. 1997;5:234-238.

2. Hersekli MA, Akpinar S, Ozalay M, Ozkoc G, Cesur N, Uysal M, Pourbagher A, Tandogan RN. Tunnel enlargement after arthroscopic anterior cruciate ligament reconstruction: comparison of bone-patellar tendon-bone and hamstring autografts. Adv Ther. 2004;21:123-131.

3. Clatworthy MG, Annear P, Bulow J-U, Bartlett RJ. Tunnel widening in anterior cruciate ligament reconstruction: a prospective evaluation of hamstring and patella tendon grafts. Knee Surgery, Sport Traumatol Arthrosc. 1999;7:138-145.

4. Biswal UK, Balaji G, Nema S, Poduval M, Menon J, Patro DK. Correlation of tunnel widening and tunnel positioning with short-term functional outcomes in single-bundle anterior cruciate ligament reconstruction using patellar tendon versus hamstring graft: a prospective study. Eur J Orthop Surg Traumatol. 2016;26:647-655.

5. Fauno $P$, Kaalund $S$. Tunnel widening after hamstring anterior cruciate ligament reconstruction is influenced by the type of graft fixation used: A prospective randomized study. Arthrosc J Arthrosc Relat Surg. 2005;21:1337-1341.

6. Mathis DT, Rasch H, Hirschmann MT. In vivo bone tunnel remodeling in symptomatic patients after ACL reconstruction: a retrospective comparison of articular and extra-articular fixation. Muscles Ligaments Tendons J. 2016;5:316-324.

7. Nurmi JT. Porcine Tibia Is a Poor Substitute for Human Cadaver Tibia for Evaluating Interference Screw Fixation. Am J Sports Med. 2004;32:765-771.

8. Tudisco C, Bisicchia S, Cosentino A, Chiozzi F, Piva M. Knee stability, athletic performance and sport-specific tasks in nonprofessional soccer players after ACL reconstruction: comparing trans-tibial and antero-medial portal techniques. Muscles Ligaments Tendons J. 2015;5:175-180.

9. Martin SD, Martin TL, Brown $\mathrm{CH}$. Anterior cruciate ligament graft fixation. Orthop Clin North Am. 2002;33:685-696.

10. Woo SL-Y, Hollis JM, Adams DJ, Lyon RM, Takai S. Tensile properties of the human femur-anterior cruciate ligament-tibia complex: The effects of specimen age and orientation. Am J Sports Med. 1991;19:217-225.

11. Cooper DE, Deng XH, Burstein AL, Warren RF. The strength of the central third patellar. tendon graft: A biomechanical study. Am J Sports Med. 1993;21:818-824.

12. Stäubli HU, Schatzmann L, Brunner P, Rincón L, Nolte LP. Quadriceps tendon and patellar ligament: cryosectional anatomy and structural properties in young adults. Knee Surg Sports Traumatol Arthrosc. 1996;4:100-110.

13. Kousa $P$, Järvinen TLN, Vihavainen $M$, Kannus $P$, Järvinen $M$. The fixation strength of six hamstring tendon graft fixation devices in anterior cruciate ligament reconstruction. Part II: tibial site. Am J Sports Med. 2003;31:182-188.

14. Milano G, Mulas PD, Ziranu F, Piras S, Manunta A, Fabbriciani C, Brand J, Weiler A, Caborn DN, Brown CH, et al. Comparison Between Different Femoral Fixation Devices for ACL Reconstruction With Doubled Hamstring Tendon Graft: A Biomechanical Analysis. Arthrosc J Arthrosc Relat Surg. 2006;22: 660-668.

15. Krupp R, Scovell F, Cook C, Nyland J, Wyland D. Femoral Cross-Pin Safety in Anterior Cruciate Ligament Reconstruction as a Function of Femoral Tunnel Position and Insertion Angle. Arthrosc J Arthrosc Relat Surg. 2011;27:83-88.

16. McGlaston TJ, Entezari V, Nazarian A, Ramappa AJ. The Safe Zone for TransFix Fixation in Anterior Cruciate Ligament Reconstruction Using the Anteromedial Portal Technique. Arthrosc J Arthrosc Relat Surg. 2011;27:77-82.

17. Gougoulias N, Khanna A, Griffiths D, Maffulli N. ACL reconstruction: Can the transtibial technique achieve optimal tunnel positioning? A radiographic study. Knee. 2008;15:486-490. 
18. Kanazawa T, Soejima T, Noguchi K, Tabuchi K, Noyama M, Nakamura K-I, Shiba N. Tendon-to-bone healing using autologous bone marrow-derived mesenchymal stem cells in ACL reconstruction without a tibial bone tunnel-A histological study. Muscles Ligaments Tendons J. 2014;4:201-206.

19. Colvin A, Sharma C, Parides M, Glashow J. What Is the Best Femoral Fixation of Hamstring Autografts in Anterior Cruciate Ligament Reconstruction? A Meta-analysis. Clin Orthop Relat Res. 2010;469:1075-1081.

20. Lovric V, Kanazawa T, Nakamura Y, Oliver RA, Yu Y, Walsh WR. Effects of Gaps Induced Into the ACL Tendon Graft on Tendon-Bone Healing in a Rodent ACL Reconstruction Model. Muscles Ligaments Tendons J. 2011;1:91-99.

21. Maak TG, Voos JE, Wickiewicz TL, Warren RF. Tunnel Widening in Revision Anterior Cruciate Ligament Reconstruction. Am Acad Orthop Surg. 2010;18:695-706.

22. Xu Y, Ao Y, Wang J, Yu J, Cui G. Relation of Tunnel Enlargement and Tunnel Placement After Single-Bundle Anterior Cruciate Ligament Reconstruction. Arthrosc J Arthrosc Relat Surg. 2011;27:923-932.

23. Rittweger J, Maffulli N, Maganaris CN, Narici MV. Reconstruction of the anterior cruciate ligament with a patella-tendonbone graft may lead to a permanent loss of bone mineral content due to decreased patellar tendon stiffness. Med Hypotheses. 2005;64:1166-1169.

24. Weber AE, Delos D, Oltean HN, Vadasdi K, Cavanaugh J, Potter HG, Rodeo SA. Tibial and Femoral Tunnel Changes After ACL Reconstruction: A Prospective 2-Year Longitudinal MRI Study. Am J Sports Med. 2015;43:1147-1156.

25. Padulo J, Oliva F, Frizziero A, Maffulli N. Muscles, Ligaments and Tendons Journal - Basic principles and recommendations in clinical and field Science Research: 2016 Update. MLTJ. 2016;6(1):1-5.

26. Baumfeld JA, Diduch DR, Rubino LJ, Hart JA, Miller MD, Barr MS, Hart JM. Tunnel widening following anterior cruciate ligament reconstruction using hamstring autograft: a comparison between double cross-pin and suspensory graft fixation. Knee Surg Sports Traumatol Arthrosc. 2008;16:1108-1113.

27. Kong C-G, In Y, Kim G-H, Ahn C-Y. Cross Pins versus Endobutton Femoral Fixation in Hamstring Anterior Cruciate Ligament Reconstruction: Minimum 4-Year Follow-Up. Knee
Surg Relat Res. 2012;24:34-39.

28. Lui PPY, Lee YW, Mok TY, Cheuk YC. Peri-tunnel bone loss: does it affect early tendon graft to bone tunnel healing after ACL reconstruction? Knee Surgery, Sport Traumatol Arthrosc. 2013;23:740-751.

29. Janssen RPA, Scheffler SU. Intra-articular remodelling of hamstring tendon grafts after anterior cruciate ligament reconstruction. Knee Surgery Sport Traumatol Arthrosc. 2013;22: 2102-2108.

30. Magnussen RA, Binzel K, Zhang J, Wei W, Flanigan DC, Hewett T, Kaeding CC, Knopp M. Anterior cruciate ligament graft metabolic activity assed by PET-MRI. 2016;312.

31. Baksh N, Hannon CP, Murawski CD, Smyth NA, Kennedy JG. Platelet-Rich Plasma in Tendon Models: A Systematic Review of Basic Science Literature. Arthrosc J Arthrosc Relat Surg. 2013;29:596-607.

32. Mayer C, Magnussen RA, Servien E, Demey G, Jacobi M, Neyret P, Lustig S. Patellar Tendon Tenodesis in Association With Tibial Tubercle Distalization for the Treatment of Episodic Patellar Dislocation With Patella Alta. Am J Sports Med. 2012;40:346-351.

33. Matsumoto T, Kuroda R, Matsushita T, Araki D, Hoshino Y, Nagamune K, Kurosaka M. Reduction of Tunnel Enlargement With Use of Autologous Ruptured Tissue in Anterior Cruciate Ligament Reconstruction: A Pilot Clinical Trial. Arthrosc J Arthrosc Relat Surg. 2014;30:468-474.

34. Mutsuzaki H, Sakane M. Calcium phosphate-hybridized tendon graft to enhance tendon-bone healing two years after ACL reconstruction in goats. BMC Sport Sci Med Rehabil. $2011 ; 3$.

35. Lui PPY, Lee YW, Mok TY, Cheuk YC. Local administration of alendronate reduced peri-tunnel bone loss and promoted graft-bone tunnel healing with minimal systemic effect on bone in contralateral knee. J Orthop Res. 2013;31:1897-1906.

36. Cerulli G, Zamarra G, Vercillo F, Pelosi F. ACL reconstruction with "the original all-inside technique." Knee Surgery, Sport Traumatol Arthrosc. 2011;19:829-831.

37. Ko YW, Rhee SJ, Kim IW, Yoo J-D. The Correlation of Tunnel Position, Orientation and Tunnel Enlargement in Outside-in Single-Bundle Anterior Cruciate Ligament Reconstruction. Knee Surg Relat Res. 2015;27:247-54. 